\title{
Magnetization and Inter-Filament Contact in HEP and ITER Bronze-Route $\mathrm{Nb}_{3} \mathrm{Sn}$ Wires
}

\author{
B. Bordini, D. Bessette, L. Bottura, A. Devred, M. Jewell, D. Richter, and C. Senatore
}

\begin{abstract}
Magnetization measurements are relevant tests for the characterization of superconductors. Practically they are the only measurements that allow estimating the critical current density at low fields of low temperature superconductors, the effective filament size and the hysteresis losses. For this purpose CERN, in collaboration with the University of Geneva, has carried out magnetization measurements on five types of $\mathrm{Nb}_{3} \mathrm{Sn}$ wires: three bronze route strands used in the ITER project; one Powder In Tube (PIT) and one Internal Tin (IT) wires used for developing next generation accelerator magnets. The field dependent magnetization has been determined using three setups: a Vibrating Sample Magnetometer (VSM), a Superconducting Quantum Interference Device (SQUID) and a special system used for the production control of LHC strands. Samples of different lengths have been tested to check the different coupling between the filaments. Unexpectedly, it was found that the magnetization of the tested bronze wires was strongly dependent on the sample length. In this paper, the results, which were obtained for different type of strands and sample lengths, are reported and compared.
\end{abstract}

Index Terms-Inter-filament contact, length effect, magnetization, $\mathrm{Nb}_{3} \mathrm{Sn}$.

\section{INTRODUCTION}

$\mathbf{M}$ AGNETIZATION measurements are practically the only test that allow estimating the hysteresis losses and the effective filament size of superconductors and, the critical current density at low fields of low temperature superconductors. Furthermore magnetization measurements are essential for characterizing multi-filamentary $\mathrm{Nb}_{3} \mathrm{Sn}$ wires, especially when they are used in magnets that require limited magnetic hysteresis losses and high field quality. Indeed multi-filamentary $\mathrm{Nb}_{3} \mathrm{Sn}$ wires often exhibit magnetic hysteresis losses that are larger than what one can estimate considering the dimension of the $\mathrm{Nb}_{3} \mathrm{Sn}$ filaments and the superconductor critical current. The excess losses and magnetization are due to inter-filament coupling giving rise to an effective filament size that can be significantly larger than the actual filament size. The coupling can be due to different effects: 1) eddy currents in the matrix, that occurs when the conductor is exposed to changing fields; 2 ) the proximity effect, where the thin layer of copper between filaments gets superconducting at internal fields close to $0 \mathrm{~T} ; 3$ ) bridging between the filaments, generally occurring in high $J_{c}$

Manuscript received August 01, 2010; accepted September 28, 2010. Date of publication December 23, 2010; date of current version May 27, 2011.

B. Bordini, L. Bottura, and D. Richter are with the Technology Department, CERN, Geneva 23, 1211 CH Switzerland (e-mail: bernardo.bordini@cern.ch).

D. Bessette, A. Devred, and M. Jewell are with ITER International Organization, Saint-Paul-Lez-Durance, 13108, France.

C. Senatore is with the University of Geneva, Geneva, Switzerland.

Color versions of one or more of the figures in this paper are available online at http://ieeexplore.ieee.org.

Digital Object Identifier 10.1109/TASC.2010.2092733
TABLE I

STRAND PROPERTIES

\begin{tabular}{cccccc}
\hline \hline $\begin{array}{c}\text { Strand } \\
\text { Number }\end{array}$ & $\begin{array}{c}\text { Strand } \\
\text { Type }\end{array}$ & Technology & $\begin{array}{c}\text { Diameter, } \\
(\mathrm{mm})\end{array}$ & $\begin{array}{c}\text { Pitch, } \\
(\mathrm{mm})\end{array}$ & $\begin{array}{c}\text { Density } \\
\left(\mathrm{gr} / \mathrm{cm}^{3}\right)\end{array}$ \\
\hline 1 & ITER & Bronze & 0.83 & 15 & 8.71 \\
2 & ITER & Bronze & 0.83 & 15 & 9.05 \\
3 & ITER & Bronze & 0.82 & 15 & 8.82 \\
4 & RRP 54/61 & Internal Tin & 0.80 & 12 & 8.46 \\
5 & PIT 192 & Powder & 1.00 & 24 & - \\
\hline \hline
\end{tabular}

internal tin strands [1]-[3]; 4) inter-filament contact, observed in Internal Tin strands [4]. The eddy current effect is time dependent and it decays in time; all the other three effects are time-independent and cannot be prevented by slowing the field ramp rates. The filament coupling can be reduced by decreasing the twist pitch in the case it is generated by eddy currents [5], by the proximity effect [6] and, by the inter-filament contact [4]. Furthermore the filament coupling due to these 3 effects depends on the sample length: a short sample has an effective twist pitch equal to the sample length [4], [5].

Recently the CERN superconducting laboratory launched a campaign of tests on different type of state of the $\operatorname{art~}^{N_{3}} \mathrm{Sn}_{3} \mathrm{Sn}$ strands in order to investigate the effect on the magnetization measurements of the sample length. Five types of strands were measured for this study: 3 different bronze-route (candidates for the ITER magnets), 1 Internal Tin (IT) by Oxford Superconducting Technology (OST) and 1 Powder In Tube (PIT) by Bruker EAS. These last 2 strands are, at present, the most suitable conductor on the market for building next generation accelerator magnets. The field dependent magnetization has been determined using three setups: a Vibrating Sample Magnetometer (VSM), a Superconducting Quantum Interference Device (SQUID) and a special system used for the production control of LHC strand. Unexpectedly, it was found that the magnetization of the tested bronze wires is strongly dependent on the sample length. In this paper the results are reported and compared.

\section{SAMPLE PREPARATION}

Five different strands were considered for this study, the main characteristics of such conductors are summarized in Table I. All the strands were measured in the fixed coil magnetometer at CERN; furthermore some of these strands were also tested at the University of Geneva: strands 1 and 2 (see Table I) in a VSM; strands 3 and 4 in a SQUID.

For the samples measured at CERN, $20 \mathrm{~cm}$ long straight strands were reacted and then a single strand was inserted into a 
glass tube ( $\sim 3.2 \mathrm{~mm}$ outer diameter, $\sim 1.2 \mathrm{~mm}$ inner diameter) that was finally filled with epoxy resin. Some of the samples were tested in this configuration (named 'CERN-18 cm' samples: although the sample length is $20 \mathrm{~cm}$, the pick-up coils of the CERN magnetometer can measure a maximum length of $18 \mathrm{~cm}$ ), others were cut in pieces of a certain length that, after having been electrically insulated at the end with epoxy resin, were then reassembled in $20 \mathrm{~cm}$ long tubes. In particular two piece lengths were investigated $1.5 \mathrm{~cm}$ and $0.5 \mathrm{~cm}$. The samples constituted by these pieces were respectively named 'CERN-1.5 cm' and 'CERN-0.5 cm' samples.

For the measurement in the VSM and in the SQUID, $10 \mathrm{~cm}$ long straight wires were reacted and then cut in samples about $0.5 \mathrm{~cm}$ long. For the SQUID, tightly packed mini-coils (the adjacent turns almost touched each other) were wound on a stainless steel tube approximately $3.8 \mathrm{~mm}$ in diameter. During the heat treatment, the coils were fixed to the steel tube through to screws at the ends of the strand. After the heat treatment the mini coils were removed from the support and cut in samples of different lengths ( $\sim 1$ turn, $\sim 2.5$ turns, $\sim 7.5$ turns).

All the samples were heat treated in vacuum atmosphere. The RRP strand was reacted at CERN while all the others at the University of Geneva. The 3 ITER bronze-route strands were heat treated with a final plateau of $650{ }^{\circ} \mathrm{C}$ for $100 \mathrm{hrs}$; strands 1 and 2 were reacted together while strand 3 had a slightly different heat treatment (different intermediate plateaus). The RRP strand heat treatment had 3 temperatures plateaus at $205^{\circ} \mathrm{C}, 400{ }^{\circ} \mathrm{C}$ and $695^{\circ} \mathrm{C}$ lasting respectively 72,48 and $17 \mathrm{hrs}$; all the temperature ramps were carried out at $50^{\circ} \mathrm{C} / \mathrm{hr}$. Finally the PIT heat treatment had one temperature plateaus at $625^{\circ} \mathrm{C}$ lasting $250 \mathrm{hrs}$ and all the temperature ramps were carried out at $50^{\circ} \mathrm{C} / \mathrm{hr}$.

\section{Measurements Results}

All the magnetization measurements were carried in liquid helium at $4.2 \mathrm{~K}$. In this paper the magnetization $M(\mathrm{~A} / \mathrm{m})$ is reported in $\mathrm{mT}\left(1000 \cdot \mu_{0} M\right)$ and it is referred per total wire volume. In the CERN magnetometer, the field was swept between 0 and $1.45 \mathrm{~T}$ with different sweeping rates ranging from $6 \mathrm{mT} / \mathrm{s}$ to $46 \mathrm{mT} / \mathrm{s}$. In the VSM the magnetization was measured during bipolar field sweeps between $\pm 9 \mathrm{~T}$; the measurements were also repeated during bipolar field sweeps between $\pm 3 \mathrm{~T}$. Finally in the SQUID bipolar field sweeps were carried out between $\pm 3 \mathrm{~T}$ on bronze-route samples and between $\pm 4.5 \mathrm{~T}$ on the RRP samples (the RRP 54/61 suffers of severe flux-jumping at fields lower than $3 \mathrm{~T}$ ).

\section{A. ITER Bronze-Route Strands}

The magnetization of strands 1 and 2 was measured on $18 \mathrm{~cm}$, $1.5 \mathrm{~cm}$ and $0.5 \mathrm{~cm}$ straight samples at CERN and on $0.5 \mathrm{~cm}$ straight samples at UniGe. The reproducibility of the measurement was checked by testing, for each of the two strands (Samples 1 and 2, see Table I), two $18 \mathrm{~cm}$ samples at CERN and two $0.5 \mathrm{~cm}$ straight samples at UniGe.

The measurement at CERN showed a significant dependence of the magnetization on the sample length: samples shorter than the strand twist pitch have a magnetization that is significantly lower than that one of 'long' samples (length > twist pitch). The measurement results were not depending on the different

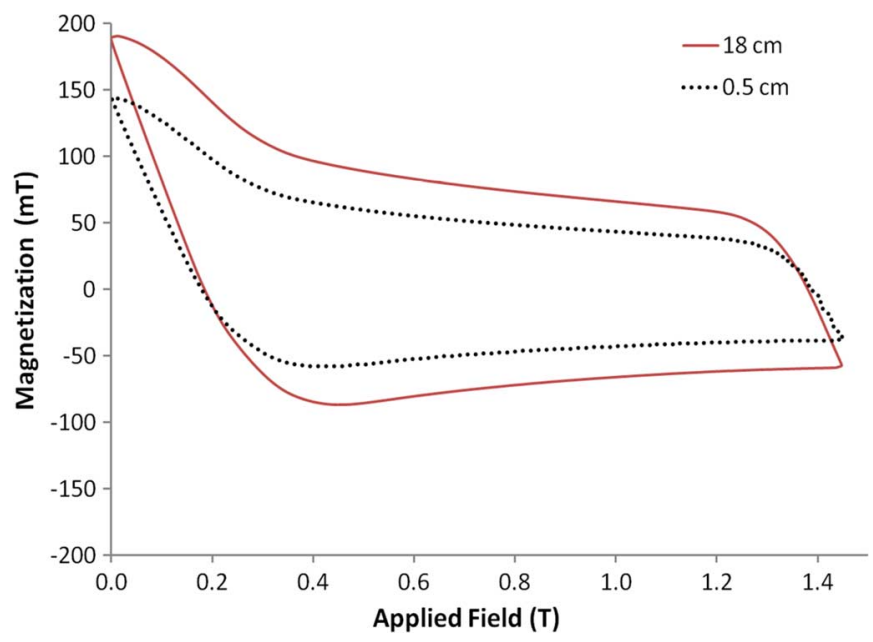

Fig. 1. Magnetization $(M)$, measured at $4.2 \mathrm{~K}$ and reported in $\mathrm{mT}$ $\left(1000 \cdot \mu_{0} M\right)$, as a function of applied field for the bronze-route strand 1 . Measurement carried out at CERN. on samples of different lengths.

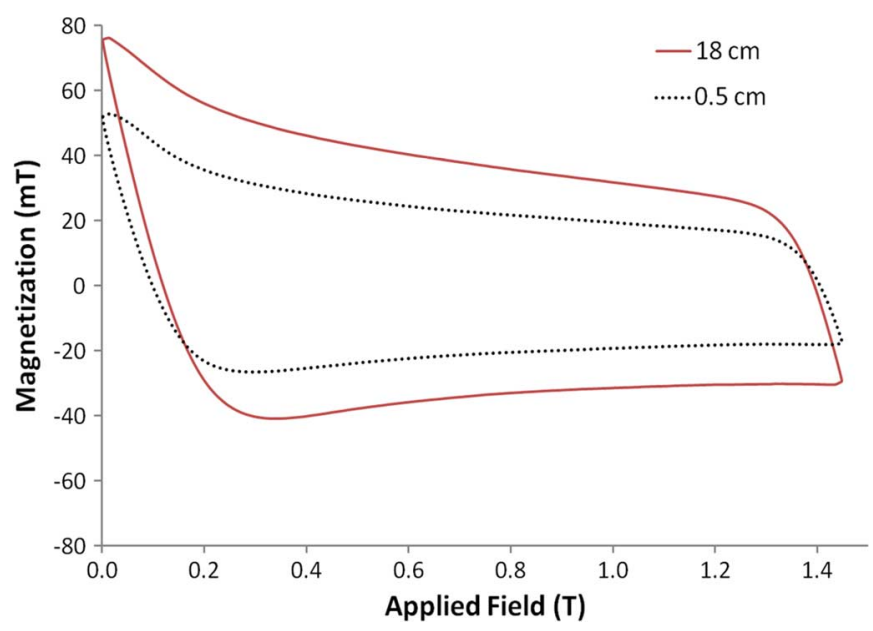

Fig. 2. Magnetization at $4.2 \mathrm{~K}$ as a function of applied field for bronze-route strand 2. Measurement carried out at CERN on samples of different lengths.

ramp rate. Figs. 1 and 2 shows the magnetization cycle measured at CERN on $18 \mathrm{~cm}$ samples and on a $0.5 \mathrm{~cm}$ samples for the strands 1 and 2 respectively. Comparing the width of the hysteresis loops $\left(\Delta M=M_{u p}-M_{\text {down }}\right)$ at $1 \mathrm{~T}$ one can note that the $0.5 \mathrm{~cm}$ sample has a magnetization that is more than $30 \%$ lower than the magnetization of the long sample. The width of the hysteresis loops at $1 \mathrm{~T}$ and its relative value with respect to $18 \mathrm{~cm}$ samples is summarized in Table II for all the measured ITER strands. During the sweeps from $1.45 \mathrm{~T}$ to $0 \mathrm{~T}$ the ratio between the magnetization values of sample with different lengths did not change significantly (neglecting the transition region $\sim 1.45 \mathrm{~T}-1.2 \mathrm{~T}$ where the distribution of the persistent currents is changing because of the inversion of the field sweep). The large difference in magnetization between strands 1 and 2 is due to the use of a pure $\mathrm{Nb}$ barrier around the multi-filamentary region in the Hitachi strand, vs the Ta barrier used in the strand 2. Indeed the $\mathrm{Nb}_{3}$ Sn filament size and the $\mathrm{J}_{\mathrm{c}}$ of the two strands are similar.

The measurement in the VSM on $0.5 \mathrm{~cm}$ long samples (see Fig. 3 and Table II) confirmed the results obtained at CERN for samples of similar length. The width of the hysteresis loops 
TABLE II

MAGNETIZATION AMPLITUDE OF BRONZE STRANDS

\begin{tabular}{cc|ccc|c}
\hline \hline \multicolumn{2}{c|}{ Tested at } & \multicolumn{3}{c|}{ CERN } & UniGe \\
\multicolumn{2}{c|}{ Sample Length $(\mathrm{cm})$} & 18 & $\sim 1.5$ & $\sim 0.5$ & $\sim 0.5$ \\
\hline Strand 1 & & 132 & 117 & 86 & $78^{*}$ \\
Strand 2 & $\Delta \mathrm{M}(1 \mathrm{~T})$, & 63 & 54 & 39 & $32^{*}$ \\
Strand 3 & $(\mathrm{mT})$ & 85 & 76 & 58 & $61^{* *}$ \\
\hline Strand 1 & & 1.00 & 0.89 & 0.65 & 0.59 \\
Strand 2 & $\Delta \mathrm{M} \mathrm{(1} \mathrm{T)/}$ & 1.00 & 0.86 & 0.62 & 0.51 \\
Strand 3 & $\Delta \mathrm{M}_{18 \mathrm{~cm}}(1 \mathrm{~T})$ & 1.00 & 0.89 & 0.68 & 0.75 \\
\hline \hline
\end{tabular}

$*$ VSM, ${ }^{* *}$ SQUID

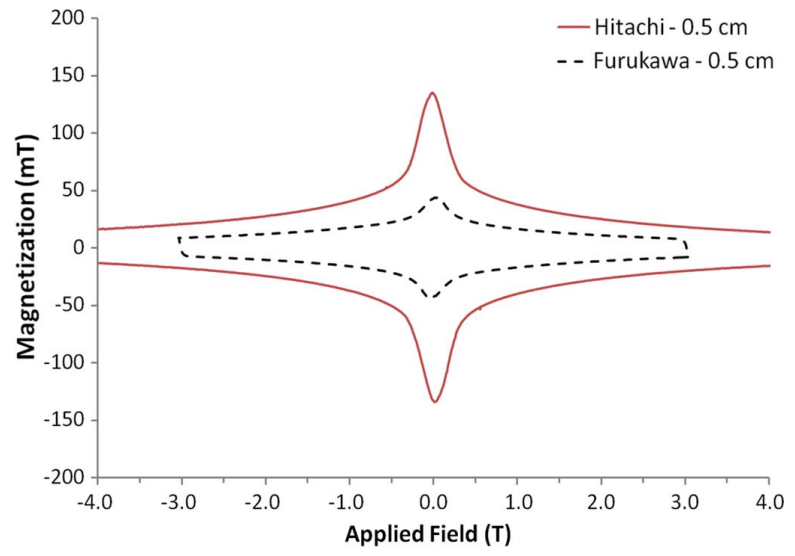

Fig. 3. Magnetization at $4.2 \mathrm{~K}$ as a function of applied field for strands 1 and 2 . Measurement carried out in a VSM at UniGe using for each sample two bipolar field sweeps $\pm 9 \mathrm{~T}$ and $\pm 3 \mathrm{~T}$.

measured in the VSM during bipolar field sweeps between $\pm 9 \mathrm{~T}$ were further processed to deduce loss integrals using the following formula:

$$
Q\left(B_{\max }\right)=2 \int_{0}^{B_{\max }} \Delta M d B
$$

which provides a good approximation of the hysteresis loss in the case of negligible contribution of the filament penetration (that is certainly the case for this type of strands with bipolar field cycles of $3 \mathrm{~T}$ amplitude; see strand 2 in Fig. 3). Table III summarizes the loss results obtained for bipolar field cycles of $3 \mathrm{~T}$ amplitude on $0.5 \mathrm{~cm}$ straight samples and also reports the values estimated on long samples. The loss estimate for long samples has been calculated by multiplying the losses measured on $0.5 \mathrm{~cm}$ straight samples with the ratio $\Delta M(1 \mathrm{~T}) / \Delta M_{18} \mathrm{~cm}(1 \mathrm{~T})$, see Table II. This estimate is based on the assumption that the ratio between the width of the hysteresis loops cycle for $0.5 \mathrm{~cm}$ long samples and the amplitude for long samples is constant by varying the field.

The strand 3 measured at CERN showed a dependence of the magnetization on the sample length (see Fig. 4) comparable to that one observed in strands 1 and 2 (see Table II). In order to crosscheck this effect using a different magnetometer, four samples of different lengths were tested in the SQUID: one 0.49 $\mathrm{cm}$ long sample was obtained from a reacted straight strand and three samples $(1.63 \mathrm{~cm}, 3.44 \mathrm{~cm}$ and, $10.5 \mathrm{~cm}$ long) from a
TABLE III

HYSTERESIS LOSSES $( \pm 3 \mathrm{~T})$ OF BRONZE STRANDS

\begin{tabular}{cc|cc}
\hline \hline & & $\begin{array}{c}\text { Measured on 5 mm } \\
\text { long Samples }\end{array}$ & $\begin{array}{c}\text { Estimated on } \\
\text { 'long' Samples }\end{array}$ \\
\hline Strand 1 & & 370 & 630 \\
Strand 2 & $\begin{array}{c}\text { Losses, } \\
\left(\mathrm{mJ} / \mathrm{cm}^{3}\right)\end{array}$ & 150 & 290 \\
Strand 3 & & 320 & 430 \\
\hline \hline
\end{tabular}

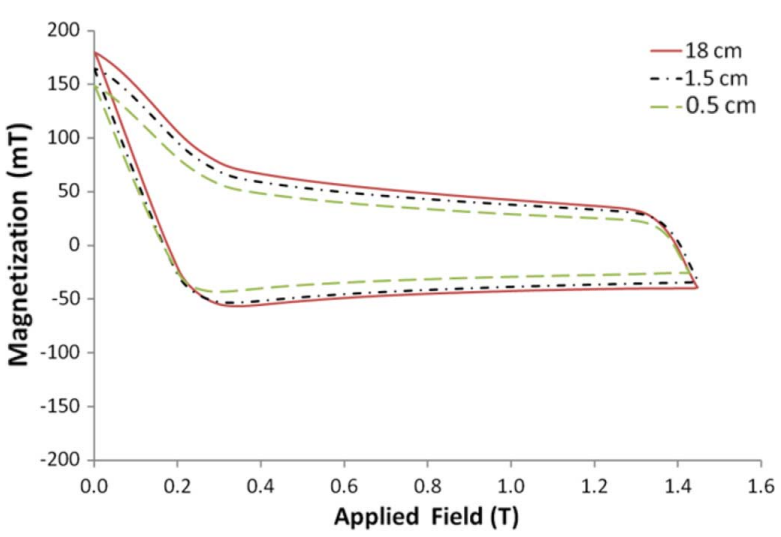

Fig. 4. Magnetization at $4.2 \mathrm{~K}$ as a function of applied field for the bronze-route strand 3. Measurement carried out at CERN on samples of different lengths.

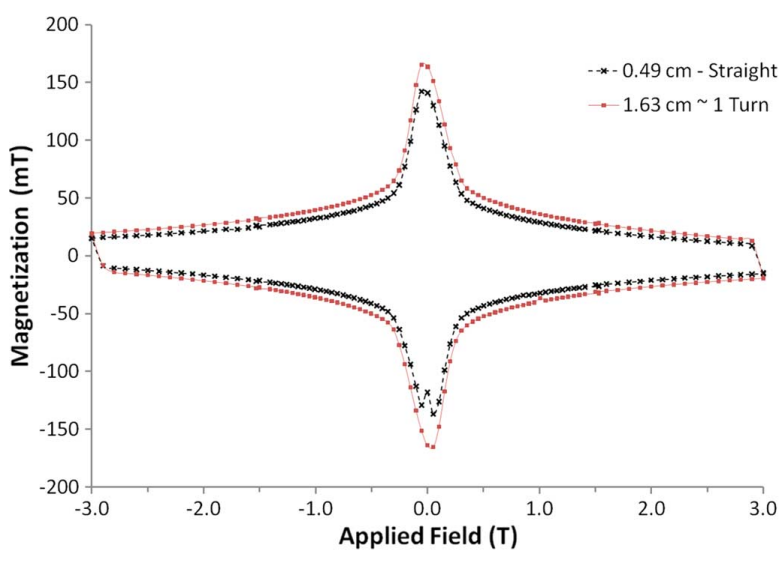

Fig. 5. Magnetization at $4.2 \mathrm{~K}$ as a function of applied field for the bronze-route strand 3. Measurement carried out in a SQUID at the University of Geneva on samples of different lengths.

reacted mini-coil (see Section II for further details). The results of the $0.49 \mathrm{~cm}$ straight sample and of the $1.63 \mathrm{~cm}$ helical sample ( $\sim 1$ turn of the mini-coil) confirmed the sample length effect on the magnetization (see Fig. 5). Furthermore, the widths of the hysteresis loops at $1 \mathrm{~T}$ of these two samples (see Table IV) are similar to those ones of the $0.5 \mathrm{~cm}$ and of the $1.5 \mathrm{~cm}$ samples tested in the fixed coil magnetometer at CERN.

It is important to remark that every measurement carried out in the SQUID with helical samples whose length was different than one turn $(1.44 \mathrm{~cm})$, produced magnetization values lower than expected. For example in Table IV: 1) the widths of the hysteresis loop of the $1.63 \mathrm{~cm}$ sample is slightly lower than that one of the $1.5 \mathrm{~cm}$ sample measured at CERN (72.5 mT instead of $76 \mathrm{mT}$ ) contrary to what observed for $0.5 \mathrm{~cm}$ straight samples, see Table II; 2) increasing the number of turn the magnetization decreases see Table IV. This behavior is most likely due to: 1 ) 
TABLE IV

MAGNETIZATION AMPLITUDE BRONZE STRAND 3

\begin{tabular}{l|cccc}
\hline \hline \multirow{2}{*}{ Sample Length } & $\begin{array}{c}0.49 \mathrm{~cm} \\
\text { Straight }\end{array}$ & $\begin{array}{c}1.63 \mathrm{~cm} \\
\sim 1 \text { Turn }\end{array}$ & $\begin{array}{c}3.44 \mathrm{~cm} \\
\sim 2.5 \text { Turns }\end{array}$ & $\begin{array}{c}10.5 \mathrm{~cm} \\
7.5 \text { Turns }\end{array}$ \\
\hline$\Delta \mathrm{M}(1 \mathrm{~T}),(\mathrm{mT})$ & 61 & 72.5 & 71.3 & 65.6 \\
\hline \hline
\end{tabular}

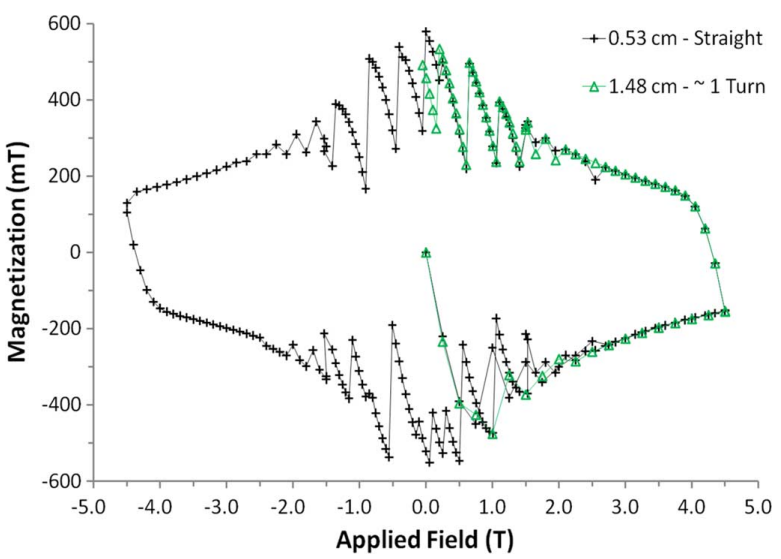

Fig. 6. Magnetization at $4.2 \mathrm{~K}$ as a function of applied field for a $0.8 \mathrm{~mm} \mathrm{RRP}$ $54 / 61$. Measurement carried out in a SQUID at the University of Geneva on samples of different lengths.

the field screening effect produced on one turn by the other turns of the coil [7], [8];2) the assumption in the measurement of a perfectly dipolar field centered in the axis of the SQUID pick up coils (this assumption in the calibration of the SQUID might underestimates the magnetization of coil samples up to $10 \%$ [4]).

From the magnetization measurement of the short straight sample in the SQUID, the strand hysteresis loss has been calculated and the results are reported in Table III.

\section{B. EAS-PIT, OST-RRP}

The $1 \mathrm{~mm}$ PIT strand measured at CERN did not show any significant sample length effect, while no conclusions could be drawn for the $0.8 \mathrm{~mm}$ RRP 54/61 because this conductor suffers large flux-jumps in the field region where the CERN fixed coil magnetometer operates.

The RRP conductor was then measured in the SQUID; two samples were tested: 1 ) a $0.53 \mathrm{~cm}$ straight sample; 2) a 1 turn sample. Longer samples could not be tested in the SQUID because of saturation problems. The measurements showed that the magnetization of the RRP strand does not increase with the length of the sample, see Fig. 6.

\section{Discussion}

The measurements on the bronze-route ITER strands showed that the magnetization of these conductors is significantly dependent on the sample length when the sample is smaller than the twist pitch of the wire. The smaller is the sample the lower is the magnetization. The measurements showed that this behavior is not dependent on the field value and on the field ramp rate, hence the coupling cannot be due to proximity effect and eddy currents in the matrix. The coupling is not even due to bridging between the filaments because that could not explain the length effect (filament bridging is not affected by the filament twisting). The only phenomenon that can explain this behavior is inter-filament contact [4] where the distance between contact points is significantly smaller than the strand twist pitch. For samples smaller than one twist pitch, the longer is the sample the larger is the number of contact points and hence the coupling between the filaments. This behavior was never observed before in bronze route wires.

Regarding the PIT and RRP conductor, the magnetization measurement did not show any significant sample length effect. That is due to the fact that each sub-element acts as a single big filament (in the RRP the filaments in each sub-element are completed merged) and the sub-elements are separated each other by a sufficiently width layer of copper $(\sim 5-10 \mu \mathrm{m})$ that keeps them decoupled.

\section{CONCLUSION}

The magnetization of five different state of the $\operatorname{art~}^{N_{3}} \mathrm{Sn}$ wires ( 3 bronze-route, one PIT and one RRP) has been measured at $4.2 \mathrm{~K}$ using 3 different magnetometers: a fixed coil magnetometer at CERN, a VSM and a SQUID at the University of Geneva. Unexpectedly it was found that the magnetization of the bronze route strands is significantly dependent on the sample length for samples smaller than one twist pitch. This phenomenon, never observed in bronze route strands, was due to inter-filament contact. That implies the use of sufficiently long samples (longer than one twist pitch) for measuring the hysteresis losses of such conductors. Regarding the PIT and RRP conductor, the magnetization measurement did not show any significant sample length effect. In these conductors each sub-element acts as a single big filament and the sub-elements are well decoupled each other.

\section{REFERENCES}

[1] A. K. Ghosh, K. E. Robins, and W. B. Sampson, "Magnetization measurements on multifilamentary $\mathrm{Nb}_{3} \mathrm{Sn}$ and $\mathrm{NbTi}$ conductors," IEEE Trans. Magn., vol. 21, pp. 328-331, Mar. 1985.

[2] R. B. Goldfarb and J. W. Ekin, "Hysteresis losses in fine filament internal-tin superconductors," Cryogenics, vol. 26, p. 478, 1986.

[3] A. K. Ghosh et al., "Magnetization studies of high $\mathrm{J}_{\mathrm{c}} \mathrm{Nb}_{3} \mathrm{Sn}$ strands," IEEE Trans. Applied Supercon., vol. 15, pp. 3494-3497, Jun. 2005.

[4] F. B. Goldfarb and K. Itoh, "Reduction of interfilament contact loss in $\mathrm{Nb}_{3} \mathrm{Sn}$ superconductor wires," J. Appl. Phys., vol. 75, no. 4, pp. 2215-2118, Feb. 15, 1994.

[5] M.-N. Wilson et al., "Experimental and theoretical studies of filamentary superconducting composites," J. Appl. Phys., vol. 3, pp. 1517-1585, Nov. 1970.

[6] W. J. Carr, Jr., "Effect of twist on wires made from in situ superconductors," J. Appl. Phys., vol. 54, pp. 6549-6552, Nov. 1983.

[7] F. Sumiyoshi, F. Irie, and K. Yoshida, "The effect of demagnetization on the eddy-current loss in a single-layered multifilamentary superconducting coil," J Appl Phys., vol. 51, pp. 3807-3811, 1980.

[8] V. B. Zenkevitch and A. S. Romanyuk, "Effect of magnetic properties of a composite superconductor on the losses in a variable magnetic field. Part 3: Collective interaction of turns," Cryogenics, vol. 20, pp. 79-85, Feb. 1980. 